\title{
Actividades de ocio y consumo abusivo de alcohol en adolescentes gallegos escolarizados
}

\section{Leisure activities and abusive consumption of alcohol in Galician adolescents}

\author{
Eliezer Pazos Millán*, José Mª Failde Garrido*, María D. Dapía Conde*, Laura Ruiz Soriano* \\ * Universidade de Vigo
}

\begin{abstract}
Resumen
En el presente estudio pretendemos analizar, en población adolescente escolarizada, la relación entre el ocio y consumo de alcohol, en función del género. En este sentido, hemos planteado un estudio cuasi-experimental de corte transversal. Mediante un muestreo multietápico, estratificado por provincia, género, tipo de centro y curso, con una muestra de 1532 participantes. Los resultados evidencian la importancia que el estilo de ocio tiene sobre el consumo y abuso de alcohol. En él, se aprecian diferencias en función del género en la prevalencia e intensidad de consumo. Estos datos son de utilidad, para el diseño e implementación de acciones preventivas.

Palabras clave: adolescentes, ocio, alcohol, tabaco, género.
\end{abstract}

\begin{abstract}
In this study we intend to analyze, in adolescent school population, the relation between leisure time and alcohol consumption, according to gender. In this sense, we have proposed a quasi-experimental cross-sectional study. Using a multistage sampling, stratified by province, gender, kind of school and grade, with a sample of 1532 participants. The results show the importance that the style of leisure has on the consumption and abuse of alcohol. In it, gender differences in prevalence and intensity of consumption are seen. These data are useful for the design and implementation of preventive actions.

Keywords: adolescent, leisure, alcohol, tabacco, gender.
\end{abstract}

\section{Introducción}

Existe una alta relación entre el consumo de sustancias y los estilos de ocio. Esta relación es especialmente significativa en el caso de los adolescentes. Así, múltiples estudios documentan como la relación con distintas sustancias adictivas se produce fundamentalmente en contextos vinculados al ocio. Los resultados generales de la muestra indican que los participantes invierten el $48 \%$ de su ocio en actividades que se realizan fuera de casa

Si nos centramos en el ocio nocturno, encontramos que un $19,2 \%$ de chicos y un $19,9 \%$ de chicas frecuentan como mínimo varias veces al mes bares, discotecas o pubs $(\chi 2=16,29, p<, 012)$, porcentajes que aumentan con la edad $\left(27,2 \%\right.$ en $4^{\circ}$ de E.S.O.). Un $18,7 \%$ de chicos y un $16,3 \%$ de chicas asisten con frecuencia a discotecas o pistas de baile $(\chi 2=21,69$, $\mathrm{p}<, 001),\left(21,5 \%\right.$ para $4^{\circ}$ de E.S.O. $)$.

Por otro lado, hemos observado que un $51,8 \%$ de los chicos y un $49,4 \%$ de las chicas han consumido alcohol en algún momento $(\chi 2=1,76, p<, 415)$ y un $17.1 \%$ de los chicos $y$ un $14.7 \%$ de las chicas participan habitualmente en botellones $(\chi 2=1,49, \mathrm{p}<, 222)$; al igual que en los demás casos, estos porcentaje se incrementan considerablemente a medida que aumenta la edad (26,0\% para $4^{\circ}$ de E.S.O.). Si consideramos las participaciones puntuales obtenemos que un $31,4 \%$ de los chicos y un $34,7 \%$ de las chicas han participado alguna vez en el botellón). Si atendemos a los problemas que se generan en el ámbito del botellón observamos que existen diferencias significativas en función del género $(\mathrm{F}=30,399, \mathrm{p}<.000)$, siendo los varones los que refieren un mayor número de comportamientos problemáticos.

\section{Método}

En el presente apartado, referimos cuál ha sido el método empleado para llevar a cabo la investigación. Para ello, definimos la muestra, instrumentos y procedimiento utilizado en el estudio.

\section{Participantes}

El estudio se ha realizado con la participación de 1532 adolescentes, de los cuales se han desechado 62 por haber sido cubiertos de una forma inadecuada o por presentar respuestas inconsistentes. Finalmente se han incluido en el estudio 1470 cuestionarios de adolescentes escolarizados en los cuatro cursos de la etapa de educación secundaria obligatoria (E.S.O. en sus siglas en español). La distribución del alumnado fue de la siguiente manera $1^{\circ}$ E.S.O. 26,9\%; $2^{\circ}$ E.S.O. 26,4\%; $3^{\circ}$ E.S.O. $24,5 \%$ y $4^{\circ}$ E.S.O. $22,2 \%$, alumnos pertenecientes a las cuatro provincias de la comunidad autónoma de Galicia (A Coruña 40,3\%, Lugo 11\%, Ourense $10,5 \%$ y Pontevedra 38,2\%). Estos parámetros se han ajustado a la realidad sociodemográfica del territorio.

\section{Instrumentos}

Los instrumentos utilizados en esta investigación fueron los siguientes: 
Cuestionario de datos sociodemográficos. Fue diseñado a medida para la presente investigación. Recogía datos tales como: sexo, edad, curso, posición filial, estudios parentales, área de residencia y datos sobre sus calificaciones personales y la actitud hacia el profesorado.

Cuestionario del uso del ocio. Recoge información sobre la gestión que hacen los participantes de su tiempo de ocio. Algunas de las cuestiones son: Tiempo libre semanal, dinero del que se dispone, preferencias, control parental, horas de ocio nocturno y otros.

Sustancias y su consumo. Recaba datos del contacto que ha tenido el adolescente con diferentes sustancias. Las sustancias analizadas son: el Tabaco, Alcohol, Cannabis, Cocaína, Drogas de síntesis y alucinógenas. Se pregunta sobre la edad de inicio, consumo en los últimos 6 meses, frecuencias de consumo y durante qué actividades.

Escala de autoestima de Rosemberg (Rosemberg, 1965). Aplicada con la sintaxis revisada por Atienza, Balaguer y Moreno (2000), es una de las escalas más conocidas para medir la consideración positiva o negativa de uno mismo y del respeto propio. (Donnellan, Trzesniewski, \& Robins, 2011).

Escala de Búsqueda de sensaciones. Este parámetro se midió mediante la Escala de Búsqueda de Sensaciones para Niños y Adolescentes (EBS-J, Pérez, Ortet, Plá et al., 1987) derivada de la "Escala de Búsqueda de Sensaciones" (Sensation Seeking Scale, SSS, Zuckerman, Eysenck y Eysenck, 1978). Consta de 50 ítems que se contestan señalando "verdadero" o "falso".

\section{Procedimiento}

Los cuestionarios fueron aplicados entre los meses de octubre de 2014 y mayo de 2015, teniendo en cuenta las estadísticas de alumnado del curso anterior. Para realizar los análisis pertinentes, hemos planteado un estudio cuasi-experimental de corte transversal. Para la recogida de datos se ha utilizado un muestreo multietápico, estratificado por conglomerados. El primer estrato fue la provincia, el segundo, la titularidad del centro educativo y como tercero el ámbito donde se encontraba el centro.

A cada participante se le entregó un único documento que contenía los instrumentos de medida descritos en el apartado anterior.

Antes de entregar los cuestionarios, se les informó del carácter voluntario y anónimo de los mismos y les fue explicado el motivo de la encuesta y los objetivos que se pretendían alcanzar con la realización del estudio.

\section{Diseño}

Hemos planteado un estudio cuasi-experimental, descriptivo y transversal, mediante encuestas.

\section{Análisis de datos}

Para el análisis de datos se recurrió a la utilización de técnicas de estadística descriptiva univariada, medias y desviaciones típicas, frecuencias y porcentajes, así como contrastes analíticos mediante la prueba de chi cuadrado. Los datos fueron analizados mediante el programa estadístico SPSS, versión 24 para Windows.

\section{Resultados}

En este estudio se ha medido el tiempo de ocio en la cantidad de horas semanales que los adolescentes disponen para sí mismos o para desarrollar actividades de su elección. Los resultados demuestran que los chicos disponen de una cantidad media de horas significativamente superior a las chicas (Tabla 1), obteniendo una diferencia significativa en los tres análisis, Ocio general $(\mathrm{F}=12.942, \mathrm{p}<.000)$. Ocio en casa $(\mathrm{F}=19.749, \mathrm{p}<.000)$ y Ocio fuera de casa $(\mathrm{F}=9.569, \mathrm{p}<$ $.002)$.

Sin embargo no se han encontrado diferencias significativas, entre hombres y mujeres, en la cantidad de dinero del que disponen cada semana para invertir en su tiempo de ocio. Además cabe destacar que el 71,1\% de los chicos y el $65,6 \%$ de las chicas se sienten satisfechos con la cantidad y empleo de su tiempo de ocio, no existiendo diferencias significativas en esta cuestión.

Tabla 1.

Horas de Ocio

\begin{tabular}{lcc}
\hline & $\begin{array}{c}\text { Hombres } \\
\text { N=716 } \\
\text { Media (Sx) }\end{array}$ & $\begin{array}{c}\text { Mujeres } \\
\text { N=754 } \\
\text { Media (Sx) }\end{array}$ \\
\hline Horas de Ocio & 19,3 & 16,2 \\
Horas ocio en casa & $(17,6)$ & $(15,1)$ \\
Horas ocio fuera de casa & 11,8 & 9,3 \\
& $(11,8)$ & $(9,1)$ \\
& 10,7 & 8,9 \\
& $(11,9)$ & $(9,8)$ \\
\hline
\end{tabular}

En relación a los datos obtenidos en la sobre el tiempo dedicado a las diferentes actividades (Hombres Mujeres) encontramos que la mayor parte de las chicas invierte la mayor parte de su tiempo en actividades como salir a reunirse con los amigos $(13,7 \%-17,3 \%)$; Escuchar música, CDs, ... $(9,1 \%-22,4 \%)$ y leer libros $(2,2 \%-5,8 \%)$; mientras que los chicos prefieren invertir el tiempo de ocio en actividades deportivas $(27,5 \%$ $11,8 \%)$; Ver la televisión $(12,5 \%-9,4 \%)$ o jugar a videojuegos $(7,1 \%$ - 04\%). Aunque como podemos observar todas las actividades tienen un grado de preferencia tanto en los chicos como en las chicas.

Algunas de estas actividades coinciden con aquellas durante las que se produce algún tipo de consumo de alcohol, tabaco o cannabis, que son las tres sustancias más consumidas alguna vez, por los adolescentes de estas edades (alcohol 50,6\%; tabaco 29,9\%; cannabis $10,4 \%)$. 
Tabla 2.

Actividades habituales con mayor consumo

\begin{tabular}{|c|c|c|c|c|}
\hline $\begin{array}{l}\text { Estar con los } \\
\text { amigos }\end{array}$ & $\begin{array}{c}\text { Hombres } \\
\mathrm{N}=716 \\
\mathrm{f}(\%)\end{array}$ & $\begin{array}{c}\text { Mujeres } \\
\mathrm{N}=754 \\
\mathrm{f}(\%)\end{array}$ & $\chi^{2}$ & Sig. \\
\hline Alcohol & $\begin{array}{c}37 \\
(5,2)\end{array}$ & $\begin{array}{c}22 \\
(2,9)\end{array}$ & \multirow{3}{*}{18,17} & \multirow{3}{*}{.017} \\
\hline Tabaco & $\begin{array}{c}25 \\
(3,5)\end{array}$ & $\begin{array}{c}50 \\
(6,6)\end{array}$ & & \\
\hline Cannabis & $\begin{array}{c}9 \\
(1,3)\end{array}$ & $\begin{array}{c}6 \\
(0,8)\end{array}$ & & \\
\hline $\begin{array}{l}\text { Estar con mi } \\
\text { pareja }\end{array}$ & $\begin{array}{c}\text { Hombres } \\
\mathrm{N}=716 \\
\mathrm{f}(\%)\end{array}$ & $\begin{array}{c}\text { Mujeres } \\
\mathrm{N}=754 \\
\mathrm{f}(\%)\end{array}$ & & \\
\hline Alcohol & $\begin{array}{c}16 \\
(2,2)\end{array}$ & $\begin{array}{c}13 \\
(1,7)\end{array}$ & \multirow{3}{*}{7,82} & \multirow{3}{*}{,252 } \\
\hline Tabaco & $\begin{array}{c}18 \\
(2,5)\end{array}$ & $\begin{array}{c}37 \\
(4,9)\end{array}$ & & \\
\hline Cannabis & $\begin{array}{c}4 \\
(0,6)\end{array}$ & $\begin{array}{c}5 \\
(0,7)\end{array}$ & & \\
\hline Escuchar música & $\begin{array}{c}\text { Hombres } \\
\mathrm{N}=716 \\
\mathrm{f}(\%)\end{array}$ & $\begin{array}{c}\text { Mujeres } \\
\mathrm{N}=754 \\
\mathrm{f}(\%)\end{array}$ & & \\
\hline Alcohol & $\begin{array}{c}9 \\
(1,3)\end{array}$ & $\begin{array}{c}3 \\
(0,4)\end{array}$ & \multirow{3}{*}{16,41} & \multirow{3}{*}{, 012} \\
\hline Tabaco & $\begin{array}{c}13 \\
(1,8)\end{array}$ & $\begin{array}{c}27 \\
(3,6)\end{array}$ & & \\
\hline Cannabis & $\begin{array}{c}3 \\
(0,4)\end{array}$ & $\begin{array}{c}5 \\
(0,7)\end{array}$ & & \\
\hline
\end{tabular}

En relación al análisis de los consumos en estas actividades, observamos que para la mayor parte de los adolescentes no es una práctica habitual consumir sustancias durante su realización. En la Tabla 2 podemos ver que existen diferencias significativas en el consumo de alcohol y tabaco. Tanto en el tiempo que están con los amigos, como en el tiempo que pasan escuchando música, los chicos son los que más bebidas alcohólicas ingieren, mientras que las chicas superan a sus homólogos varones en el consumo de tabaco.

Como se puede observar, el ocio nocturno casi no se refleja en los datos citados anteriormente, puesto que a estas edades, la falta de total autonomía y la carencia de unos ingresos propios hacen que este tipo de ocio se realice con poca frecuencia.
Tabla 3.

Actividades de ocio nocturno

\begin{tabular}{|c|c|c|c|c|}
\hline Ir a discotecas, Bailar & $\begin{array}{c}\text { Hombres } \\
\mathrm{N}=716 \\
\mathrm{f}(\%)\end{array}$ & $\begin{array}{c}\text { Mujeres } \\
\mathrm{N}=754 \\
\mathrm{f}(\%)\end{array}$ & $\chi^{2}$ & Sig. \\
\hline Una vez al mes o menos & $\begin{array}{c}160 \\
(22,5)\end{array}$ & $\begin{array}{c}220 \\
(29,4)\end{array}$ & \multirow{3}{*}{16.29} & \multirow{3}{*}{.012} \\
\hline Varias veces al mes & $\begin{array}{c}67 \\
(9,4)\end{array}$ & $\begin{array}{c}100 \\
(13,4)\end{array}$ & & \\
\hline $\begin{array}{l}\text { Varias veces a la } \\
\text { semana }\end{array}$ & $\begin{array}{c}71 \\
(9,8) \\
\end{array}$ & $\begin{array}{c}49 \\
(6,5) \\
\end{array}$ & & \\
\hline Ir a bares de copas & $\begin{array}{c}\text { Hombres } \\
\mathrm{N}=716 \\
\mathrm{f}(\%)\end{array}$ & $\begin{array}{c}\text { Mujeres } \\
\mathrm{N}=754 \\
\mathrm{f}(\%)\end{array}$ & $\chi^{2}$ & Sig. \\
\hline Una vez al mes o menos & $\begin{array}{l}128 \\
(18)\end{array}$ & $\begin{array}{c}170 \\
(22,7)\end{array}$ & \multirow{3}{*}{21.69} & \multirow{3}{*}{.001} \\
\hline Varias veces al mes & $\begin{array}{c}65 \\
(9,1)\end{array}$ & $\begin{array}{c}78 \\
(10,4)\end{array}$ & & \\
\hline $\begin{array}{l}\text { Varias veces a la } \\
\text { semana }\end{array}$ & $\begin{array}{c}68 \\
(9,6) \\
\end{array}$ & $\begin{array}{c}44 \\
(5,9)\end{array}$ & & \\
\hline Ir a conciertos & $\begin{array}{c}\text { Hombres } \\
\mathrm{N}=716 \\
\mathrm{f}(\%)\end{array}$ & $\begin{array}{c}\text { Mujeres } \\
\mathrm{N}=754 \\
\mathrm{f}(\%)\end{array}$ & & \\
\hline Una vez al mes o menos & $\begin{array}{c}241 \\
(33,8)\end{array}$ & $\begin{array}{l}338 \\
(45)\end{array}$ & \multirow{3}{*}{9.35} & \multirow{3}{*}{.155} \\
\hline Varias veces al mes & $\begin{array}{c}40 \\
(5,6)\end{array}$ & $\begin{array}{c}25 \\
(3,3)\end{array}$ & & \\
\hline $\begin{array}{l}\text { Varias veces a la } \\
\text { semana }\end{array}$ & $\begin{array}{c}71 \\
(9,9)\end{array}$ & $\begin{array}{c}36 \\
(4,7)\end{array}$ & & \\
\hline
\end{tabular}

Referente al ocio nocturno, las actividades que realizan con más frecuencia son: Ir a discotecas o salas de baile, ir a bares de copas o asistir a conciertos de diferente índole (ver Tabla 3).

En estas actividades obtenemos diferencias significativas, tanto en la cantidad de adolescentes de educación secundaria obligatoria que frecuentan bares de copas, como en los que acceden a discotecas o salas de baile. En ambas situaciones existe un mayor número de chicas que practican este tipo de ocio. 
Tabla 4.

Consumo de sustancias adictivas durante las actividades de ocio nocturno

\begin{tabular}{|c|c|c|c|c|}
\hline Ir a discotecas, Bailar & $\begin{array}{c}\text { Hombres } \\
\mathrm{N}=716 \\
\mathrm{f}(\%)\end{array}$ & $\begin{array}{c}\text { Mujeres } \\
N=754 \\
\mathrm{f}(\%)\end{array}$ & $\chi^{2}$ & Sig. \\
\hline Alcohol & $\begin{array}{c}124 \\
(17,4)\end{array}$ & $\begin{array}{c}167 \\
(22,2)\end{array}$ & \multirow{3}{*}{9.46} & \multirow{3}{*}{.222} \\
\hline Alcohol y tabaco & $\begin{array}{c}28 \\
(3,9)\end{array}$ & $\begin{array}{c}38 \\
(5,1)\end{array}$ & & \\
\hline $\begin{array}{l}\text { Alcohol, tabaco y } \\
\text { cannabis }\end{array}$ & $\begin{array}{c}16 \\
(2.2) \\
\end{array}$ & $\begin{array}{c}13 \\
(1,7) \\
\end{array}$ & & \\
\hline Ir a bares de copas & $\begin{array}{c}\text { Hombres } \\
\mathrm{N}=716 \\
\mathrm{f}(\%)\end{array}$ & $\begin{array}{c}\text { Mujeres } \\
\mathrm{N}=754 \\
\mathrm{f}(\%)\end{array}$ & & \\
\hline Alcohol & $\begin{array}{c}152 \\
(21,3)\end{array}$ & $\begin{array}{c}165 \\
(22,0)\end{array}$ & \multirow{3}{*}{10.30} & \multirow{3}{*}{172} \\
\hline Alcohol y tabaco & $\begin{array}{c}31 \\
(4,3)\end{array}$ & $\begin{array}{c}58 \\
(7,7)\end{array}$ & & \\
\hline $\begin{array}{l}\text { Alcohol, tabaco y } \\
\text { cannabis }\end{array}$ & $\begin{array}{c}17 \\
(2.4) \\
\end{array}$ & $\begin{array}{c}17 \\
(2.3) \\
\end{array}$ & & \\
\hline Ir a conciertos & $\begin{array}{c}\text { Hombres } \\
\mathrm{N}=716 \\
\mathrm{f}(\%)\end{array}$ & $\begin{array}{c}\text { Mujeres } \\
\mathrm{N}=754 \\
\mathrm{f}(\%)\end{array}$ & & \\
\hline Alcohol & $\begin{array}{l}47 \\
(6.6)\end{array}$ & $\begin{array}{c}51 \\
(6.8)\end{array}$ & \multirow{3}{*}{4.80} & \multirow{3}{*}{.778} \\
\hline Alcohol y tabaco & $\begin{array}{c}10 \\
(1,4)\end{array}$ & $\begin{array}{c}14 \\
(1,9)\end{array}$ & & \\
\hline $\begin{array}{l}\text { Alcohol, tabaco y } \\
\text { cannabis }\end{array}$ & $\begin{array}{c}8 \\
(1,1) \\
\end{array}$ & $\begin{array}{c}4 \\
(0,5) \\
\end{array}$ & & \\
\hline
\end{tabular}

Como era de esperar, la prevalencia de consumo durante las actividades de ocio nocturno son más elevadas que en las actividades habituales (como se observa en la tabla 4), sin embargo, no se han encontrado diferencias significativas de consumo de alcohol o de otras sustancias entre los chicos y las chicas en estas actividades.

\section{Botellón}

Uno de los temas más polémicos del ocio juvenil, en España durante los últimos años ha sido el botellón, esta práctica consiste en reuniones juveniles nocturnas (casi siempre multitudinarias) en las que se busca socializar con otros jóvenes, se consumen diversas sustancias y se permite cierto nivel de desinhibición (Calafat, Juan, Becoña, et. Al., 2005). Por ello hemos decidido tratarlo como un tema aparte.

Los datos reflejan que el $31,7 \%$ de los chicos y el $34,7 \%$ de las chicas de esta etapa educativa han participado activamente en el botellón, sin encontrar diferencias significativas en función del género $(\mathrm{F}=1,45$, $p=, 229)$. A su vez se han encontrado diferencias significativas en la frecuencia de asistencia $(F=10,97$, $p=, 027$ ) habiendo un mayor número de chicas que han asistido 2 veces en los últimos 6 meses $(53,6 \%$ de hombres $-66 \%$ de mujeres) y presentando un número más elevado de chicos $(31,2 \%)$ que de chicas $(19,7 \%)$ en la frecuencia de una o varias veces al mes.
Tabla 5.

4Consumo en el botellón

\begin{tabular}{|c|c|c|c|c|}
\hline Alcohol & $\begin{array}{c}\text { Hombres } \\
\mathrm{N}=227 \\
\mathrm{f}(\%)\end{array}$ & $\begin{array}{c}\text { Mujeres } \\
\mathrm{N}=261 \\
\mathrm{f}(\%)\end{array}$ & $\mathrm{F}$ & Sig. \\
\hline $1-2$ veces & $\begin{array}{c}52 \\
(23,7)\end{array}$ & $\begin{array}{c}67 \\
(27,0)\end{array}$ & \multirow{3}{*}{9,79} & \multirow{3}{*}{.044} \\
\hline $3-5$ veces & $\begin{array}{c}19 \\
(8,6)\end{array}$ & $\begin{array}{c}41 \\
(16,3)\end{array}$ & & \\
\hline Más de 5 veces & $\begin{array}{c}101 \\
(45,9)\end{array}$ & $\begin{array}{c}104 \\
(41,3)\end{array}$ & & \\
\hline Tabaco & $\begin{array}{c}\text { Hombres } \\
\mathrm{N}=716 \\
\mathrm{f}(\%)\end{array}$ & $\begin{array}{c}\text { Mujeres } \\
\mathrm{N}=754 \\
\mathrm{f}(\%)\end{array}$ & & \\
\hline $1-2$ veces & $\begin{array}{c}27 \\
(12,3)\end{array}$ & $\begin{array}{c}64 \\
(25,6)\end{array}$ & \multirow{3}{*}{19,22} & \multirow{3}{*}{, 001} \\
\hline $3-5$ veces & $\begin{array}{c}10 \\
(4,5)\end{array}$ & $\begin{array}{c}18 \\
(7,2)\end{array}$ & & \\
\hline Más de 5 veces & $\begin{array}{c}54 \\
(24,5) \\
\end{array}$ & $\begin{array}{c}65 \\
(25,7) \\
\end{array}$ & & \\
\hline Cannabis & $\begin{array}{c}\text { Hombres } \\
\mathrm{N}=716 \\
\mathrm{f}(\%)\end{array}$ & $\begin{array}{c}\text { Mujeres } \\
\mathrm{N}=754 \\
\mathrm{f}(\%)\end{array}$ & & \\
\hline $1-2$ veces & $\begin{array}{c}24 \\
(10,9)\end{array}$ & $\begin{array}{c}27 \\
(10,8)\end{array}$ & \multirow{3}{*}{11,59} & \multirow{3}{*}{, 021} \\
\hline $3-5$ veces & $\begin{array}{c}11 \\
(5,0)\end{array}$ & $\begin{array}{c}11 \\
(4,4)\end{array}$ & & \\
\hline Más de 5 veces & $\begin{array}{c}35 \\
(15,9)\end{array}$ & $\begin{array}{c}19 \\
(7,6)\end{array}$ & & \\
\hline
\end{tabular}

Las razones principales de participación en esta actividad, son las mismas tanto de varones como de mujeres. Ellos aseguran participar en este evento para "Relacionarme con otra gente de mi edad" (40,5\%) y "Ahorrar dinero en copas" de establecimientos de ocio noctuno $(22,5 \%)$.

En la tabla 5, analizamos la frecuencia de consumo y la diferencia de género de las tres principales sustancias que consumen los adolescentes.

Tal como su nombre indica, el producto de mayor consumo en el botellón es el alcohol, siendo consumido en algún momento por la mayoría de los asistentes. Tal como observamos en la tabla 5 , encontramos diferencias significativas en el consumo de todas las sustancias. En relación al alcohol y al tabaco, encontramos diferencias significativas en las frecuencias más bajas, siendo un mayor número de mujeres las que consumen estas sustancias con menor frecuencia.

En cuanto al cannabis, también existen diferencias significativas, pero esta vez en las frecuencias altas de consumo, donde hay un número más elevado de varones que consumen con mayor frecuencia esta sustancia.

\section{Conclusiones}

Si bien el rango de edades de este estudio es habitualmente monitorizado en los estudios de prevanlencia tales como la encuesta ESTUDES, llevada a cabo por el Plan Nacional de Drogas, es sin embargo un tema que necesita de monitoreo constante, pues se trata de conductas con alto dinamismo y cambio. 
A la luz de los datos aportados por este estudio, las actividades de ocio habituales de los adolescentes presentan un consumo bajo de cualquiera de las sustancias analizadas, aún así, se han hayado diferencias significativas en el consumo de alcohol y tabaco, siendo el primero más consumido por los varones, mientras que el tabaco es más consumido por las mujeres durante las actividades de ocio cotidianas.

Cuando nos referimos específicamente a ocio nocturno, este tipo de consumos aumentan considerablemente, tanto en el consumo de alcohol, como de tabaco y cannabis. Por consiguiente, tal como vienen señalando diferentes estudios el consumo de sustancias recreativas (alcohol, tabaco, cannabis o cocaína) presentan una alta relación con la situación, en especial con los fines de semana y el ocio nocturno.

Mención especial requiere la práctica del botellón, lugar donde los consumos adquieren su punto álgido y se presentan diferencias significativas en la frecuencia de consumo de estas sustancias. Por los datos obtenidos y teniendo en cuenta el total de los participantes, hayan asistido o no al botellón, sabemos que el $24,58 \%$ de los varones y el $28,12 \%$ de las mujeres reconocen haber consumido alcohol en el botellón, aunque la mayor parte de las mujeres se agrupan en los parámetros de menor frecuencia de consuno. El mismo caso se da en relación al tabaco, donde el $12,71 \%$ de los varones y el $19,50 \%$ de las mujeres reconocen haber consumido tabaco. Especial relevancia tiene, que en esta actividad los consumos son de alta intensidad y en cortos períodos temporales, lo que supone un riesgo para intoxicaciones etílicas.

En el caso del Cannabis, existe un número menor de consumidores, el $9,78 \%$ de los varones y el 7,56\% de las mujeres, ha consumido cannabis en el botellón. El consumo de esta sustancia, presenta diferencias significativas entre el género, en la frecuencia de consuno, siendo los varones los que consumen esta sustancia con mayor frecuencia. No obstante, tal como ha sucedido con el alcohol y el tabaco, estamos asistiendo a un proceso de feminización del consumo de cannabis.

\section{Referencias}

Atienza, F. L., Moreno, Y., \& Balaguer, I. (2000). Análisis de la dimensionalidad de la Escala de Autoestima de Rosenberg en una muestra de adolescentes valencianos. Revista de Psicología. Universitas Tarraconensis, vol XXII (1-- 2), 29-- 42.

Calafat, A., Juan, M., Becoña, E., Castillo, A., Fernández, C., Franco, M., ... \& Ros, M. (2005). El consumo de alcohol en la lógica del botellón. Adicciones, 17(3).

Donnellan, M. B., Trzesniewski, K. H., \& Robins, R.W. (2011). Self-Esteem. Enduring issues and controversies. In: Chamorro-Premuzic, T., von Stumm, S., \& Furnhan, A. The Wiley-Blackwell handbook of individual differences. Chichester: Blackwell Publishing Ltd.; 718-746.
Pérez, J., Ortet, G., Plá, S. y Simó, S. (1987). Escala de Búsqueda de Sensaciones para Niños y Adolescentes (EBS-J). Evaluación Psicológica, 3, 283-290.

Rosenberg, M. (1965). Society and the adolescent self-- image. Princeton, NJ: Princeton University Press.

Zuckerman, M., Eysenck, S.B. y Eysenck, A. J. (1978). Sensation seeking in England and American: cross-cultural age and sex comparisons. Journal of Consulting and Clinical Psychology, 46, 139-149. 